\title{
The Metabolism of Intravenously Injected Isotopic Cholic Acid in Laennec's Cirrhosis *
}

\author{
Morton Blum $†$ and Norton Spritz $\ddagger$ \\ (From the Lipid Metabolism Laboratory of the Second [Cornell] Medical Division, Bellevue \\ Hospital, and the Department of Medicine, Cornell University Medical College, \\ New York, N.Y.)
}

Although elevated concentrations of serum bile acids have frequently been demonstrated in patients with liver disease $(2,3)$, little other information concerning the effect of hepatic impairment on the metabolism of these substances is known. Josephson (4), utilizing a nonisotopic intravenous cholic acid tolerance test, found that obstructive jaundice could be differentiated from hepatoparenchymal disease by increased retention of cholic acid in the serum 5 and 30 minutes after injection of a $0.5-\mathrm{g}$ test dose. More recently, Carey (2) showed that the ratio of concentrations of serum trihydroxy- to dihydroxy-bile acid, rather than the absolute concentrations in patients with liver disease, may provide important diagnostic and prognostic information.

In the present study, bile acid metabolism in human liver disease was further investigated after the intravenous injection of isotopically labeled cholic acid into patients with Laennec's cirrhosis and into normal subjects. Comparisons were then made of the first 60 -minute plasma clearance of radioactivity, the rate of conjugation, and the late clearance of plasma cholic acid in both groups; finally, from these data and the plasma cholic acid concentration, cholic acid pool sizes and turnover rates were calculated in the cirrhotic group.

* Submitted for publication August 13, 1965; accepted October 28, 1965.

This study was supported in part by U. S. Public Health Service grants NB-03346-04 and 2A5091. This work was presented at the meeting of the American Gastroenterology Association, Montreal, Canada, 1965, and has been published in abstract form (1).

$\dagger$ Postdoctoral fellow (1F2AM-23, 355-01), U. S. Public Health Service.

$\ddagger$ Career Investigator, Health Research Council, New York (I-128).

Address requests for reprints to Dr. Norton Spritz, Lipid Metabolism Laboratory, Second (Cornell) Medical Division, Bellevue Hospital, 1st Ave. and 26th St., New York, N. Y. 10016.
The use of isotopic bile acid had several advantages: First, it circumvented the need for amounts of test material large enough to alter bile acid physiology. Second, it permitted the detection of cholic acid and its derivatives in body fluids without the need for complex chemical measurement, purification, or both. Finally, changes in specific radioactivity permitted evaluation of bile acid kinetics.

\section{Methods}

Materials. Cholic acid-24- ${ }^{14} \mathrm{C}^{1}$ (SA $16.1 \mu \mathrm{c}$ per $\mathrm{mg}$ ) was purified before injection by preparative two-dimensional silicic acid thin layer chromatography utilizing the following solvent systems: 1) acetic acid: ethyl ether $(1: 9) ; 2)$ isoamyl acetate : propanol : propionic acid: water $(4: 4: 3: 2)$. Deoxycholic and chenodeoxycholic acids-24- ${ }^{14} \mathrm{C}^{2}$ were similarly purified. Purification of commercially available isotopic material was found to be necessary in all instances.

Tritiated cholic acid (SA $1 \mathrm{mc}$ per $\mathrm{mg}$ ) was prepared by the Wilzbach method (5) and its purity verified in the above solvent systems. ${ }^{3}$

Conjugated bile acids used as test materials were synthesized by the method of Norman (6) from the isotopic nonconjugated acids.

Florisil used in column chromatography was prepared by the method of Carroll (7).

Patients and control group. Nine patients with a clinical diagnosis of Laennec's cirrhosis, confirmed in most cases by liver biopsy, were selected for study (Table I). None was azotemic or in hepatic coma when studied, although the majority had ascites and were undergoing diuretic therapy. Age, sex, and liver function tests are listed in Table I. The control group, consisting of seven patients similar in age to the cirrhotic group, was hospitalized with no clinical or laboratory evidence of liver or biliary tract disease.

\footnotetext{
1 New England Nuclear Corp., Boston, Mass.

2 California Corp. for Biochemical Research, Los Angeles, Calif.

3 We are grateful to Dr. S. Grundy, Rockefeller University, New York, N. Y., for supplying this material.
} 


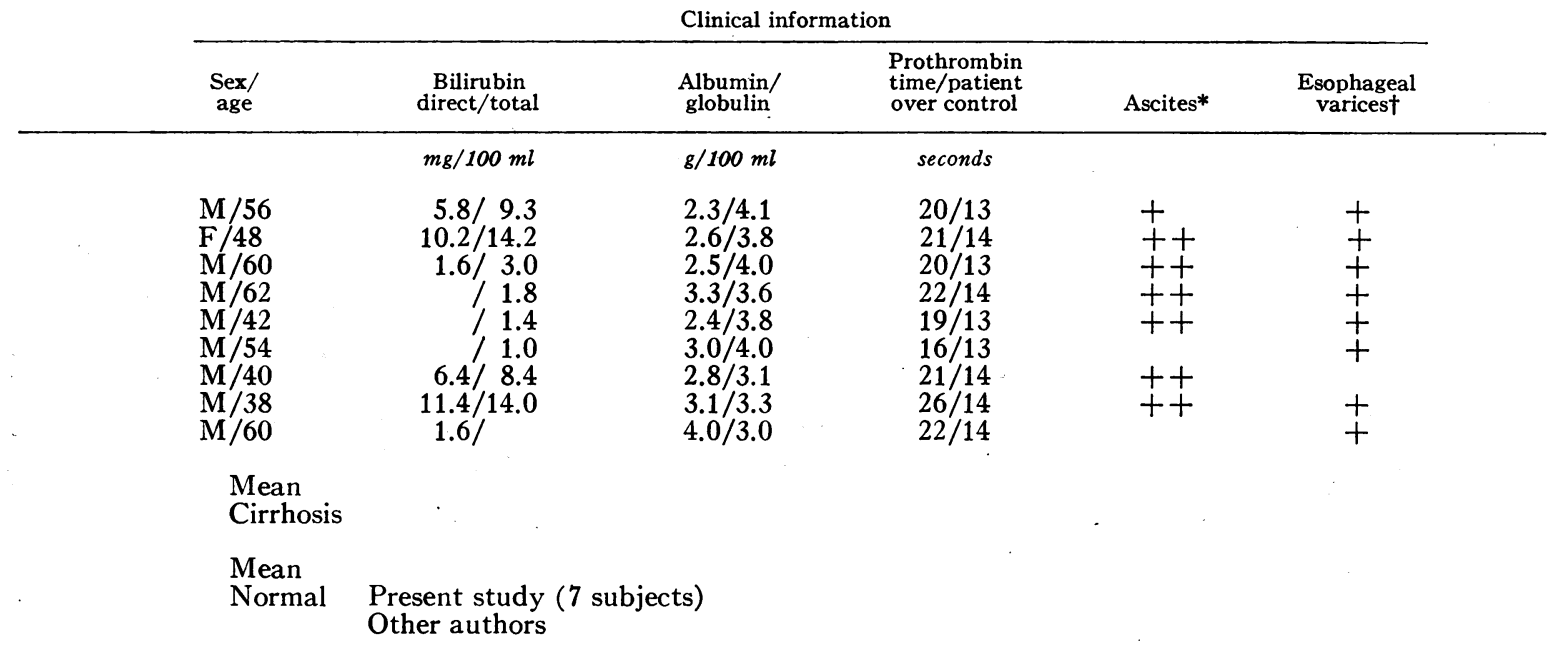

${ }^{*}+=$ physical signs without increased abdominal girth $;+=$ increased abdominal girth.

$\dagger$ By radiology or esophagoscopy.

$\ddagger t_{j}=$ half-time.

$\$$ Sandberg and associates (gas-liquid chromatography) (3).

Lindstedt (bile) (8)

I Highest value in four normal subjects.

Methods. Labeled materials were dissolved in sterile $5 \% \mathrm{NaHCO}_{8}$ solution and were sterilized by passage through an $0.8-\mu$ filter ${ }^{4}$ before intravenous injection. The dose of cholic acid-24- ${ }^{14} \mathrm{C}$ ranged from 4 to $15 \mu \mathrm{c}$, whereas two cirrhotic patients received 30 and $33 \mu \mathrm{c}$ of tritiated cholic acid. Serial plasma specimens were obtained from a vein other than the one injected $10,20,40$, and $60 \mathrm{~min}-$ utes, and then daily, after injection.

Plasma isotope fractionation. Bile acids were extracted from plasma with 5 vol of isopropyl alcohol at $65^{\circ} \mathrm{C}$ for 30 minutes. After centrifugation at $1,800 \mathrm{rpm}$ for $10 \mathrm{~min}-$ utes, the supernatant was evaporated to dryness and the residue redissolved in $3 \mathrm{ml}$ of absolute methanol. This portion was then applied to a chromatographic column of $15 \mathrm{~g}$ of Florisil. Isotope separations were carried out by a technique described in part previously (9) by utilizing the following elutions: 1) $100 \%$ ethyl ether, which elutes only neutral lipids; 2) acetic acid: ethyl ether $(1: 24)$, which elutes nonconjugated dihydroxy acids but no trihydroxy or conjugated forms; 3 ) acetic acid: ethyl ether $(1: 9)$, which elutes nonconjugated cholic acid but neither of the conjugated acids tested; and 4) acetic acid:methanol (1:9), which quantitatively elutes conjugated bile acids by using labeled taurocholic acid as a test substance. The completeness and specificity of these separations were confirmed with test solutions of repurified deoxycholic acid-24- ${ }^{14} \mathrm{C}$, chenodeoxycholic acid-24- ${ }^{14} \mathrm{C}$,

4 Millipore Corp., Bedford, Mass. cholic acid-24- ${ }^{14} \mathrm{C}$, glycodeoxycholic acid-24- ${ }^{14} \mathrm{C}$, and taurocholic acid-24- ${ }^{14} \mathrm{C}$, as shown in Table II. Fraction 1 contained less than $5 \%$ of the radioactivity and was discarded. In specimens obtained up to 60 minutes after injection of isotope, fractions 2 and 3 were pooled as the total nonconjugated fraction and the isotope in fraction 4 as the total conjugated. In specimens obtained 24 hours or longer after injection from cirrhotic subjects, the isotope was separately collected in fractions 2,3 , and 4. The bile acid components of the conjugated fraction (fraction 4) eluted with acetic acid:methanol (1:9) were identified after pressure alkaline hydrolysis (10). After acidification of the hydrolysate, the bile acids were recovered from ethyl ether in a two phase system made up of equal volumes of ethyl ether and $0.01 \mathrm{~N} \mathrm{HCl}$. The extent of the conversion of isotopic cholic acid to a dihydroxy form was then determined by measuring the relative amounts of radioactivity with the migration rates of di- and trihydroxy acids on a silicic acid thin layer chromatoplate developed with acetic acid:ethyl ether (1:9).

Determination of plasma cholic acid concentration. In specimens obtained from cirrhotic subjects, the fourth fraction eluted from the Florisil column, which contained conjugated bile acids, was analyzed chemically for cholic acid. After this fraction was subjected to pressure alkaline hydrolysis a sample of the material used for radioactivity determination was evaporated and cholic acid 
TABLE I

in nine patients with Laennec's cirrhosis

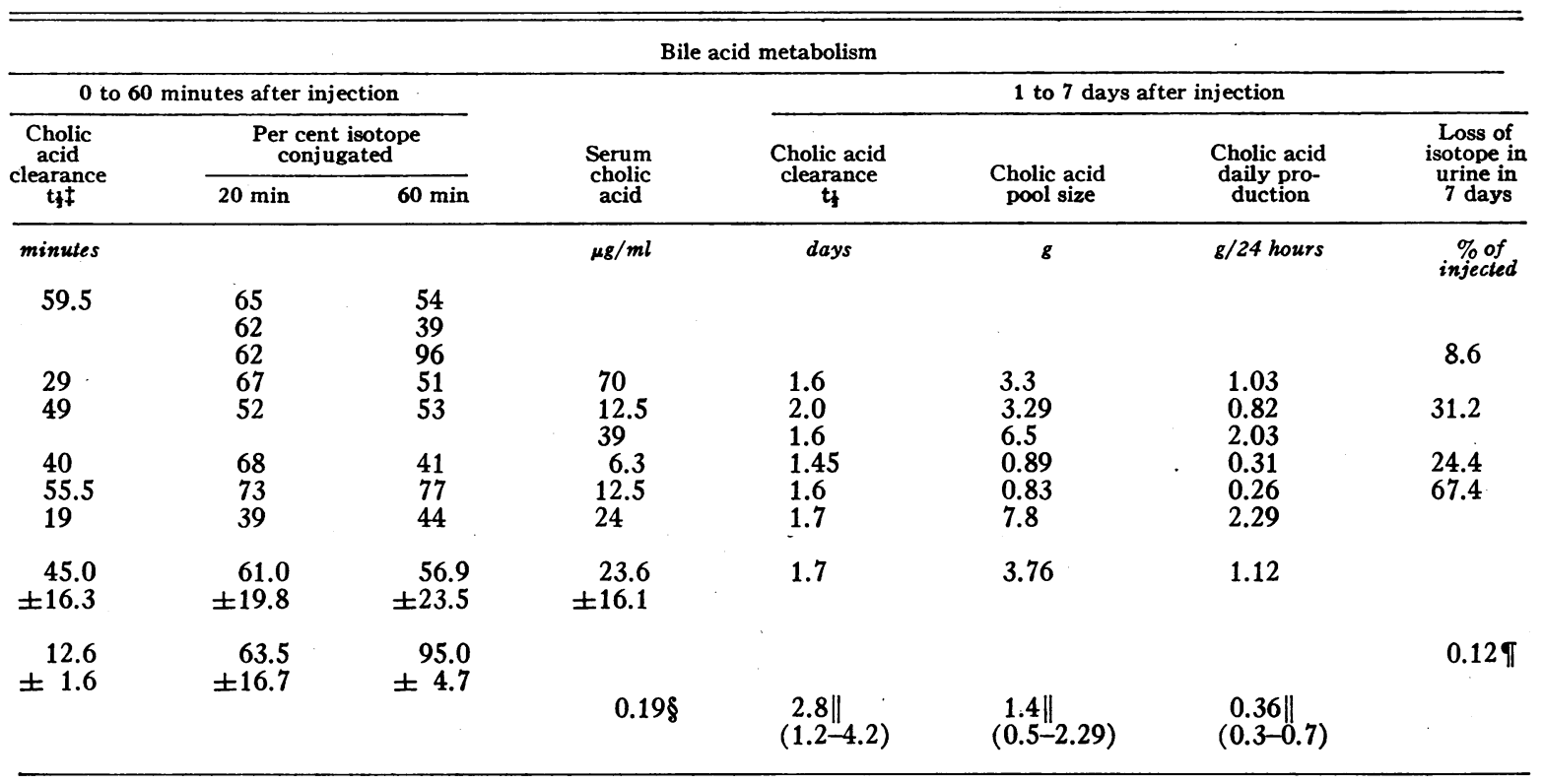

determined by the method of Mosbach, Kalinsky, Halpern, and Kendall (11). The conjugated cholic acid concentration was corrected for total isotope loss occurring during the hydrolysis procedure, and as previously reported (3) loss was as great as $40 \%$. Total plasma cholate was then calculated, assuming that the per cent of unlabeled cholic acid conjugated is the same as that of labeled cholic acid. The value presented in Table $I$ is the mean of at least two determinations 2 to 4 days after isotope injection.

\section{Results}

Initial plasma clearance of isotopic cholic acid. The rate of clearance of injected isotopic cholic acid in a group of seven normal subjects during the first 60 minutes was compared to that of eight patients with cirrhosis (Figure 1). In the normal group, the mean half time of cholic acid was $12.6 \pm 1.6$ minutes, whereas in the cirrhotic group

TABLE II

Recovery of purified isotopic test compounds from Florisil column

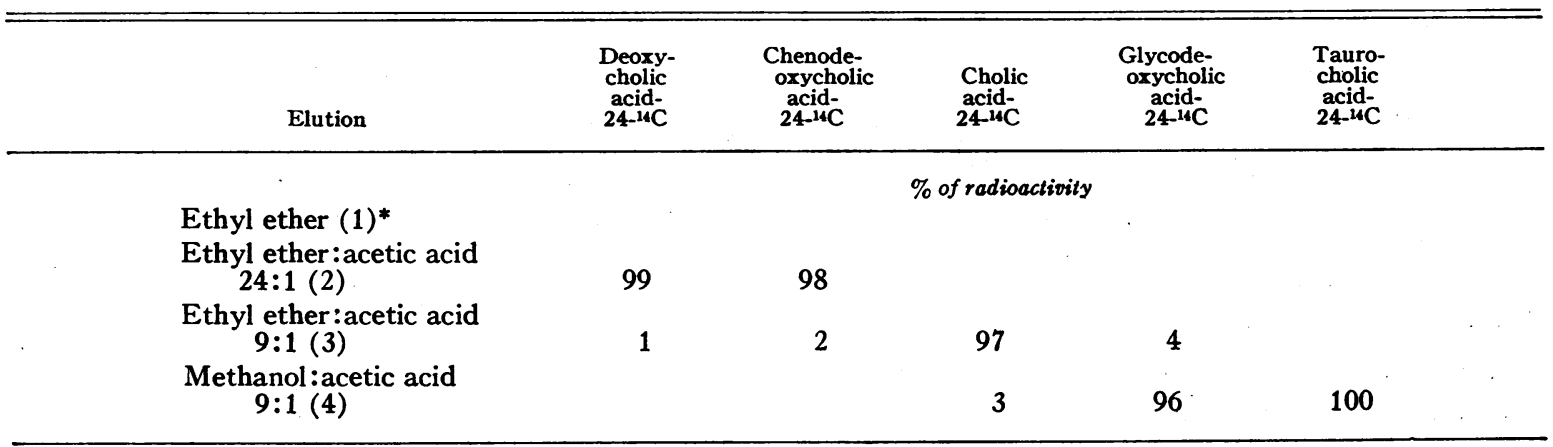

* Numbers in parentheses refer to fractions eluted (see text). 


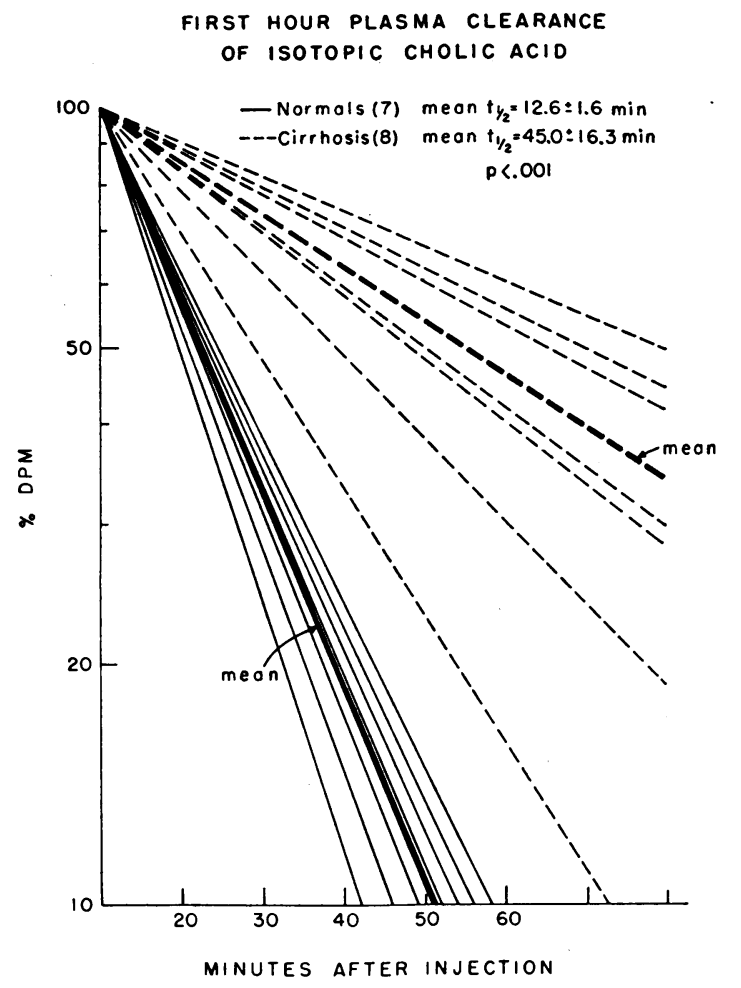

Fig. 1. Disappearance OF PLASMa ISOTOPE FOR 60 MINUTES AFTER INJECTION OF ISOTOPIC CHOLIC ACID. $t_{t}=$ half-time.

the half time was more than three times longer, averaging $45.0 \pm 16.3$ minutes. This difference was highly significant.

Per cent conjugation of plasma isotope 20 and 60 minutes after injection. In spite of the marked difference in initial clearance of radioactivity at 20 minutes, the conjugation of injected isotope in the normal and cirrhotic subjects was similar in the two groups: $63.5 \pm 16.7 \%$ and $61.0 \pm 19.8 \%$ in the normal and cirrhotic subjects, respectively. After 1 hour, however, there was a significant difference $(\mathrm{p}<0.001)$ with $56.9 \pm 23.5 \%$ conjugation in those with cirrhosis, as opposed to $95.0 \pm$ $4.7 \%$ in the normal subjects (Table III).

Plasma radioactivity 1 to 7 days after injection. In the normal subjects, plasma radioactivity was undetectable in all specimens obtained more than 6 hours after injection of isotopic cholic acid. In striking contrast, significant plasma radioactivity was demonstrable for up to 14 days in 12 cirrhotic patients (Figure 2). From 50 to $100 \%$ of this radioactivity appeared in the conjugated fraction throughout this period in the nine subjects, and when this fraction was hydrolyzed 40 to $60 \%$ was found to have been converted to a dihydroxy form.

Plasma cholic acid concentration. Plasma cholic acid concentration was measured in the conjugated bile acid fraction after hydrolysis in six cirrhotic subjects. The concentrations ranged from 6.3 to $70.0 \mu \mathrm{g}$ per $\mathrm{ml}$ with a mean of 23.6 (Table I), a figure similar to that of Sandberg, Sjövall, Sjövall, and Turner (3), who measured plasma cholic acid in cirrhotic subjects by gasliquid chromatography. These values are 30 to 140 times the mean normal value of $0.19 \mu \mathrm{g}$ per $\mathrm{ml}$ reported by those authors.

Cholic acid pool sizes and turnover rates in six cirrhotic patients. The persistence of systemic cholic acid radioactivity as well as measurable levels of plasma cholic acid content permitted the evaluation of bile acid dynamics in cirrhotic subjects without the need to sample bile or feces. Specific activity decay curves were determined from isotopic measurements in combination with chemical determination of serum cholic acid concentrations. Representative regression lines from four subjects calculated by the method of least squares (12) are shown in semilogarithmic plot in Figure 3. The points constituting the two curves that are not depicted in Figure 3 had less deviation about their calculated line than that obtained in subject A.C. and slopes within the range of the other four curves.

The mean values for half time, pool size, and calculated daily production of cholic acid were 1.7 days, $3.76 \mathrm{~g}$, and $1.12 \mathrm{~g}$, respectively (Table I). These values can be compared to similar data obtained from biliary bile acid decay curves in normal subjects reported by Lindstedt (8). The values obtained for the half time of cholic acid for

TABLE III

Per cent conjugation of plasma isotope 20 and 60 minutes after injection

\begin{tabular}{lccc}
\hline \hline & & $\begin{array}{c}\text { Per cent } \\
\text { conjugated } \\
\text { (mean } \pm \text { SD) }\end{array}$ & p \\
\hline & minutes & & \\
Cirrhosis (8) & 20 & $61.0 \pm 19.8$ & NS \\
Normal subjects (5) & 20 & $63.5 \pm 16.7$ & \\
Cirrhosis (8) & 60 & $56.9 \pm 23.5$ & $<0.001$ \\
Normal subjects (5) & 60 & $95.0 \pm 4.7$ & \\
& & &
\end{tabular}




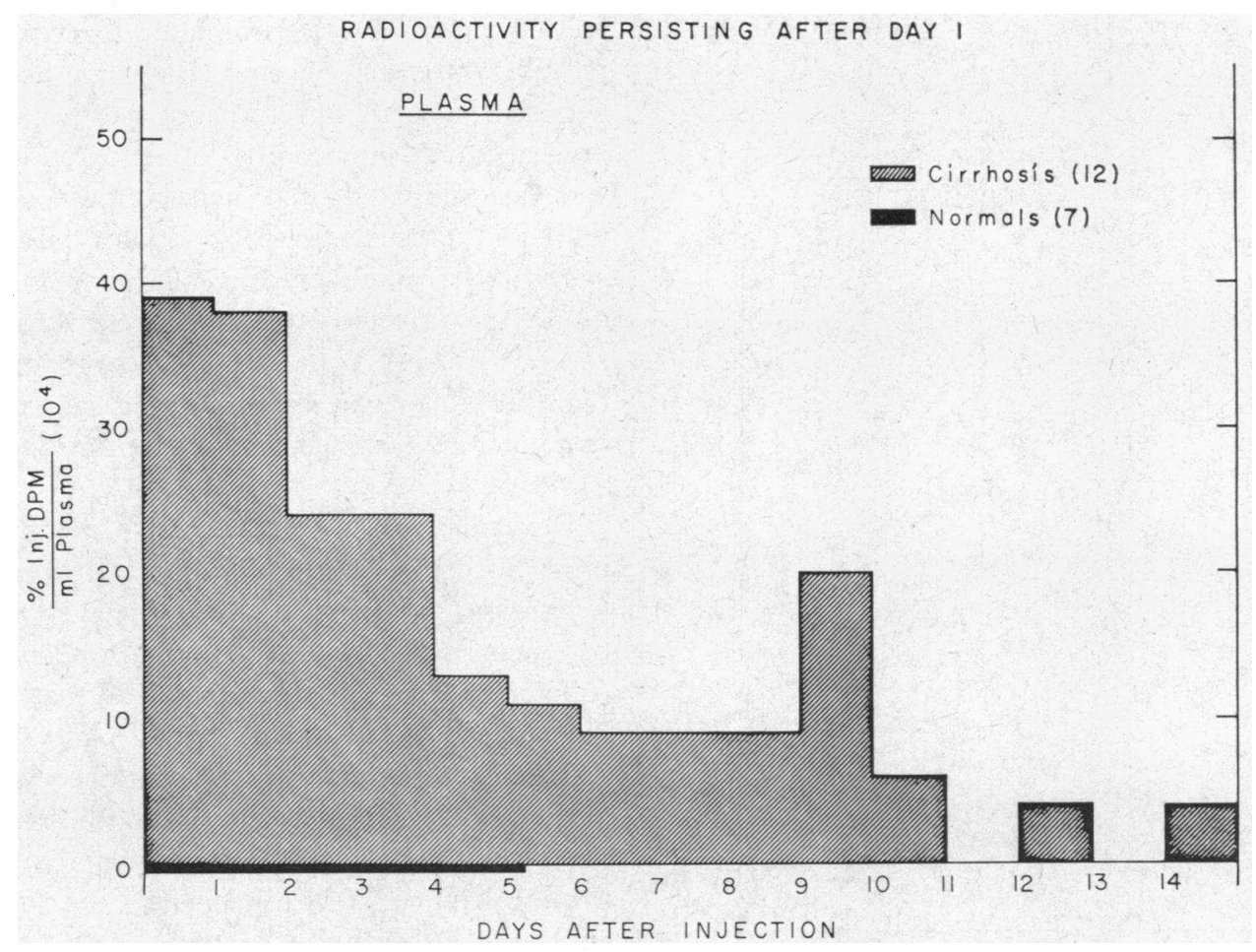

Fig. 2. Plasma Radioactivity from 1 to 14 days after injection of isotopic cholic acid.

The figures are the mean value for all specimens available on each day.

the patients in the present study were all within the range reported by that author. Pool size and daily production rate, however, exceeded the normal in four of our six subjects. These values were two to four times the highest figures reported in his study.

Urinary loss of isotope 1 to 7 days after injection. In contrast to four normal subjects in whom total isotope loss did not exceed $0.12 \%$ in 7 days, the loss in cirrhotic subjects ranged from 8.6 to $67.4 \%$ during this period. From 60 to $90 \%$ of the urinary isotope was in the conjugated form, and, as in plasma, conversion of 40 to $60 \%$ to dihydroxy forms was also noted.

Isotope in ascitic fluid. In four cirrhotic subjects isotope was detected in ascitic fluid obtained from 2 to 7 days after intravenous injection of isotopic cholic acid. By assuming that the specific.activity of plasma and ascitic fluid is the same; bile acid concentration was calculated. Ascitic cholic acid content was approximately $200 \mathrm{mg}$ in two subjects and $600 \mathrm{mg}$ in the third. These figures are based on estimates of the volume of ascites at 10 L. This represented from 10 to $25 \%$ of the cholic acid pool calculated from plasma decay curves.

\section{Discussion}

Normally in man, cholic acid is synthesized in the liver from cholesterol and excreted with bile in conjugated form into the intestine. It is then largely reabsorbed into the portal circulation, virtually none being found in the systemic blood or urine. The bile acid pool is therefore confined to the liver, bile ducts, intestine, portal circulation, and feces. The rapid and permanent disappearance from the systemic circulation of isotopically labeled cholic acid found in the present study after intravenous injection in normal subjects reflects this enterohepatic localization of the bile acid pool. The cirrhotic patient was found to handle isotopic cholic acid differently in several ways. It was cleared more slowly from the plasma, conjugated to a lesser degree, and, in greatest contrast to normal subjects, reappeared to circulate in the systemic blood for as long as 14 days.

Although in the cirrhotic group the mean decline of isotope for the first hour after injection of 


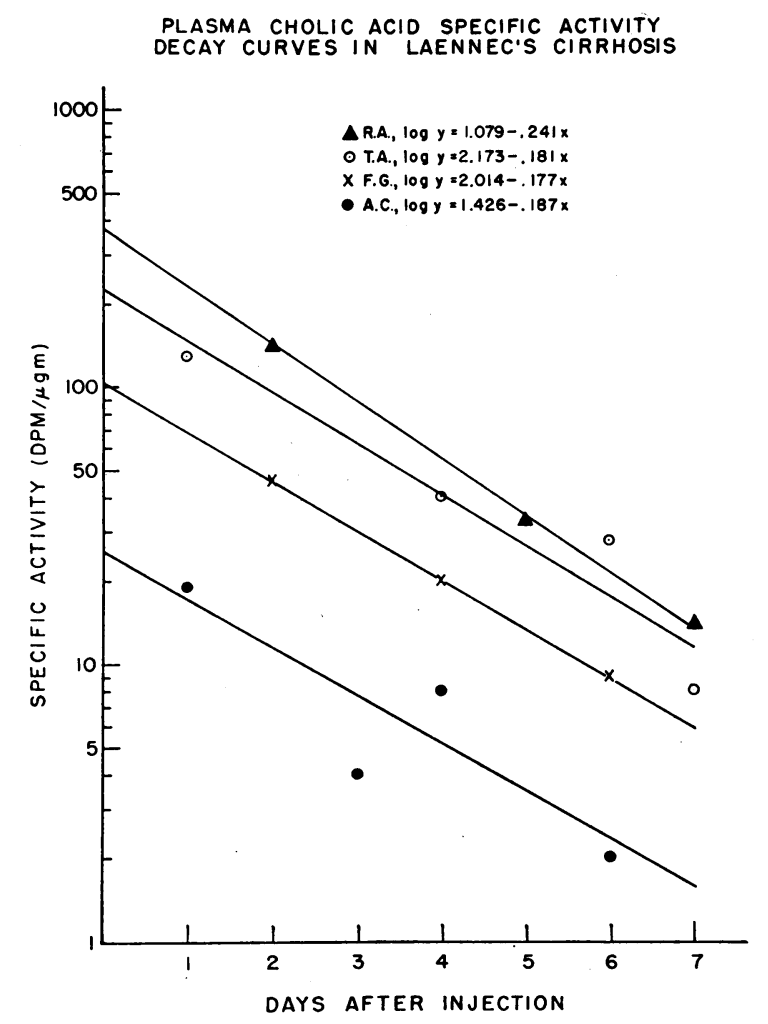

Fig. 3. Plasma ChOlic aCid SPECIFIC aCtivity DeCAy CURVES FROM DAYS 1 TO 7 AFTER INJECTION OF ISOTOPIC CHOLIC ACID IN FOUR PATIENTS WITH LAENNEC'S CIRRHOSIS.

radioactive cholic acid was about one-third that of the normal subjects, the rate of removal of cholic acid from the systemic blood into the biliary system may have been abnormally high in view of the very high plasma content of bile acid in patients with cirrhosis, ranging from tens to hundreds of times the normal shown both in the present study as well as in those previously reported $(2,3)$. The fractional disappearance rate of cholic acid was approximately $1 \%$ per minute in the normal group, so that at a mean plasma concentration of $20 \mu \mathrm{g}$ per ml, compared to 0.2 for normal subjects, actual transport rates would be more than 25 times higher in the patients with cirrhosis. The possibility also remains that differences in time required for distribution of isotope in the initial volume of distribution exist between normal and cirrhotic subjects and may play a role in the differences observed in decay rates.

Similarly, the lower per cent of isotope conjugated 60 minutes after radioactive cholic acid in- jection must also be evaluated in terms of the striking difference in circulating bile acid content between normal and cirrhotic subjects. In the present study, up to $50 \%$ of systemic cholic acid was unconjugated in the cirrhotic group so that, unlike in normal controls, a very large pool of systemic free bile acid must have been present to dilute the injected material. Therefore, the observed per cent conjugation of isotope, which is two-thirds normal, represents actual conjugation of a much larger mass of cholic acid in the cirrhotic subjects.

Regardless of the quantitative comparisons, a high per cent of isotopic cholic acid remaining in the plasma 60 minutes after injection was found to be conjugated, both in normal and in cirrhotic subjects. This finding suggests that, although biliary bile acids are essentially all conjugated (13), the rate-limiting factor in the clearance of cholic acid from the systemic blood is some process other than conjugation. This may be similar to the mechanism of clearance into the bile of bilirubin (14), but sulfobromophthalein sodium differs in that larger quantities are excreted into the bile without conjugation (15). Although the conjugated isotope observed at 60 minutes could represent material conjugated by the liver, excreted into the intestine, and reabsorbed, this possibility seems unlikely in view of the high per cent conjugation evident by 20 minutes. In normal subjects in whom conjugation was even higher, this could not be the mechanism, since reabsorption of bile acids into the systemic blood occurs only to a very small extent, certainly less than in cirrhotic patients.

In cirrhosis, the high levels of bile acids in the systemic blood, the reappearance of isotopic cholic acid, and the persistence of systemic radioactivity for as long as 14 days after the injection of radioactive cholic acid represent striking deviations from normal bile acid metabolism. Furthermore, cholic acid pool size as estimated in the present study exceeded normal values in four of six patients studied. Since fractional turnover rate was the same as normal, daily turnover was also increased. The cause of these deviations from normal is not evident from the present studies. Increased pool size is consistent with the distribution of bile acids in both the systemic and enterohepatic compartments, an abnormality that could result either from ana- 
tomical portosystemic connections or impaired bile acid extraction from portal blood by hepatic cells. In the two subjects in whom pool size was normal, however, evidences of parenchymal damage and of abnormal portosystemic connection were present. Further study is necessary to delineate the factors in cirrhosis most directly related to these bile acid abnormalities.

Similarly, the increased 24-hour turnover rate also seen in four of the six cirrhotic subjects in this study cannot be explained by the findings of this investigation. Urine loss, which does not occur in the normal subject, becomes considerable (reaching $67 \%$ in one instance) in these cirrhotic subjects as a consequence of the high systemic concentration, but the poor correlation between daily turnover of cholic acid and urine loss suggests that other mechanisms may be operative. This unaccounted loss would be fecal and the mechanism for its increase over normal, if present $(16,17)$, is not indicated from this study.

\section{Summary}

Isotopic cholic acid was injected intravenously into nine patients with Laennec's cirrhosis and into seven normal subjects. The initial removal rate was significantly depressed in cirrhosis, as was the per cent of isotope conjugated at 60 minutes. The most striking difference was the persistence of radioactivity in systemic plasma in cirrhosis for up to 14 days, compared to the permanent absence of radioactivity in normal plasma after 6 hours following injection. This persistence of radioactivity, together with the measurably high plasma cholic acid concentrations in cirrhotic subjects, permitted determination of bile acid kinetics. In four of six subjects, cholic acid pool size and turnover rate greatly exceeded values reported in normal subjects, whereas half-time for plasma falloff was within the normal range.

\section{References}

1. Blum, M., and N. Spritz. The metabolism of cholic acid-24-C $\mathrm{C}^{14}$ in Laennec's cirrhosis (abstract). Gastroenterology 1965, 48, 863.
2. Carey, J. B., Jr. The serum trihydroxy- dihydroxy bile acid ratio in liver and biliary tract disease. J. clin. Invest. 1958, 37, 1494 .

3. Sandberg, D. H., J. Sjövall, K. Sjövall, and D. A. Turner. Measurement of human serum bile acids by gas-liquid chromatography. J. Lipid Res. 1965, 6,182 .

4. Josephson, B. The circulation of the bile acids in connection with their production, conjugation and excretion. Physiol. Rev. 1943, 21, 463.

5. Wilzbach, K. E. Tritium-labeling by exposure of organic compounds to tritium gas. J. Amer. chem. Soc. 1957, 79, 1013.

6. Norman, A. Preparation of conjugated bile acids using mixed carboxylic acid anhydrides. Arkiv für Kemi 1955, 8, 331.

7. Carroll, K. K. Separation of lipid classes by chromatography on Florisil. J. Lipid Res. 1961, 2, 135.

8. Lindstedt, S. The turnover of cholic acid in man. Acta physiol. scand. 1957, 40, 1.

9. Spritz, N., and E. H. Ahrens, Jr. Direct measurement of sterols and bile acids in human feces. Biochemical Problems of Lipids (BBA Library) 1963, 1, 66.

10. Levin, S. J., J. L. Irvin, and C. G. Johnston. Spectrofluorometric determination of total bile acids in bile. Analyt. Chem. 1961, 33, 856.

11. Mosbach, E. H., J. J. Kalinsky, E. Halpern, and F. E. Kendall. Determination of deoxycholic and cholic acids in bile. Arch. Biochem. 1954, 51, 402.

12. Bailey, N. T. J. Statistical Methods in Biology. London, English Universities Press, 1959, p. 91.

13. Sjövall, J. Bile acids in man under normal and pathological conditions. Clin. chim. Acta 1960, 5, 33.

14. Schmid, R. Direct-reacting bilirubin, bilirubin glucuronide, in serum, bile, and urine. Science 1956, $124,76$.

15. Combes, B. The importance of conjugation with glutathione for sulfobromophthalein sodium (BSP) transfer from blood to bile. J. clin. Invest. 1965, 44, 1214.

16. Grundy, S. M., E. H. Ahrens, Jr., and T. A. Miettinen. Quantitative isolation and gas-liquid chromatographic analysis of total fecal bile acids. J. Lipid. Res. 1965, 6, 397.

17. Spritz, N., E. H. Ahrens, Jr., and S. Grundy. Sterol balance in man as plasma cholesterol concentrations are altered by exchanges of dietary fats. J. clin. Invest. 1965, 44, 1482. 